\title{
On Commutativity of prime gamma near rings
}

\author{
Afrah Mohammad Ibraheem \\ (Dep.of Mathematics, College of Education, Al-Mustansiriyah University, Iraq)
}

\begin{abstract}
Let $\mathrm{M}$ be a prime $\Gamma$-near ring, and let $\mathrm{F}$ and $\mathrm{G}$ be two generalized $\Gamma$-derivations of $\mathrm{M}$ with associated $\Gamma$-derivations $D_{1}$ and $D_{2}$ respectively. In this paper, we shall investigate the commutativity of $M$ by generalized $\Gamma$-derivations $\mathrm{F}$ and $\mathrm{G}$ satisfied some properties.
\end{abstract}

Key words: $\Gamma$-near ring, prime $\Gamma$-near ring, invariant set, $\Gamma$-derivation, generalized $\Gamma$-derivation.

\section{Introduction}

Satyanarayana in [6] was introduced the notion of $\Gamma$-near ring as a generalization of near-rings. The concept of $\Gamma$-derivations in $\Gamma$-near ring was introduced by Jun, Cho, and Kim [4, 2]. Asci [1] obtained some commutativity conditions for a $\Gamma$-near-ring with derivations. Some characterizations of $\Gamma$-near-rings and some regularity conditions were obtained by Cho [3]. Kazaz and Alkan [5] introduced the notion of two-sided $\Gamma$ - $\alpha$-derivation of a $\Gamma$-near-ring and investigated the commutativity of prime and semiprime $\Gamma$-near-rings. Uckun et al. [7] worked on prime $\Gamma$-near-rings with derivations and they investigated the conditions for a $\Gamma$-near-ring to be commutative. In this paper, we investigate the conditions for a prime $\Gamma$-near-ring $\mathrm{M}$ to be a commutative $\Gamma$-ring if $\mathrm{M}$ admitting a generalized $\Gamma$-derivation $\mathrm{F}$ with associated $\Gamma$-derivation $\mathrm{D}$ satisfying some properties of anon zero invariant subset Uof $\mathrm{M}$.

\section{Preliminaries}

All near-rings considered in this paper are right and left distributive.

A $\Gamma$-near-ring $\mathrm{M}$ is a triple $(\mathrm{M},+, \Gamma)$ where

(i) $(\mathrm{M},+)$ is a not necessarily abelian group,

(ii) $\Gamma$ is a non-empty set of binary operations on $\mathrm{M}$ such that for each $\alpha \in \Gamma,(\mathrm{M},+, \alpha)$ is a near-ring.

(iii) $x \alpha(y \beta z)=(x \alpha y) \beta z$ for all $x, y, z \in \mathrm{M}$ and $\alpha, \beta \in \Gamma$.

A subset $U$ of a $\Gamma$-near-ring $M$ is said to be left (resp. right) invariant if xya $\in U$ (resp. a $\gamma \mathrm{x} \in \mathrm{U}$ ), for all a $\in$ $\mathrm{U}, \gamma \in \Gamma$ and $\mathrm{x} \in \mathrm{M}$. If $\mathrm{U}$ is both left and right invariant, we say that $\mathrm{U}$ is invariant. $\mathrm{A} \Gamma$-near-ring $\mathrm{M}$ is called a prime $\Gamma$-near-ring if $\mathrm{M}$ has the property that for $\mathrm{x}, \mathrm{y} \in \mathrm{M}, \mathrm{x} \Gamma \mathrm{M} \Gamma \mathrm{y}=\{0\}$ implies $x=0$ or $y=0$. A $\Gamma$-near-ring $\mathrm{M}$ is called a semiprime if $\mathrm{M}$ has the property that for $x \in \mathrm{M}, \mathrm{x} \Gamma \mathrm{M} \Gamma \mathrm{x}=\{0\}$ implies $x=0$, and said to be 2-torsion free if for all $x \in \mathrm{M}, 2 x=0$ implies $x=0$. The centre of $\mathrm{M}$ is denoted by $\mathrm{Z}(\mathrm{M})$. For any $\mathrm{x}, \mathrm{y} \in \mathrm{M}$ and $\alpha \in \Gamma$, the notations $[\mathrm{x}, \mathrm{y}]_{\alpha}$ and $(\mathrm{xoy})_{\alpha}$ will denote $\mathrm{x} \alpha \mathrm{y}-\mathrm{y} \alpha \mathrm{x}$ and $\mathrm{x} \alpha \mathrm{y}+\mathrm{y} \alpha \mathrm{x}$, respectively .

A $\Gamma$-derivation on $M$ is defined to be an additive endomorphism $D$ of $M$ satisfying the product rule $D(x \alpha y)=$ $\mathrm{x} \alpha \mathrm{D}(\mathrm{y})+\mathrm{D}(\mathrm{x}) \alpha \mathrm{y}$, for all $\mathrm{x}, \mathrm{y} \in \mathrm{M}$ and $\alpha \in \Gamma$, or equivalently,

$\mathrm{D}(\mathrm{x} \alpha \mathrm{y})=\mathrm{D}(\mathrm{x}) \alpha \mathrm{y}+\mathrm{x} \alpha \mathrm{D}(\mathrm{y})$ for all $\mathrm{x}, \mathrm{y} \in \mathrm{N}, \alpha \in \Gamma$. The generalized $\Gamma$-derivation $\mathrm{F}$ associated with the $\Gamma$ derivation $\mathrm{D}$ is an additive endomorphism $\mathrm{F}: \mathrm{M} \rightarrow \mathrm{M}$ satisfying $\mathrm{F}(\mathrm{x} \alpha \mathrm{y})=\mathrm{x} \alpha \mathrm{F}(\mathrm{y})+\mathrm{D}(\mathrm{x}) \alpha \mathrm{y}$, for all $\mathrm{x}, \mathrm{y} \in \mathrm{M}$ and $\alpha \in \Gamma$, or equivalently, $\mathrm{F}(\mathrm{x} \alpha \mathrm{y})=\mathrm{F}(\mathrm{x}) \alpha \mathrm{y}+\mathrm{x} \alpha \mathrm{D}(\mathrm{y})$ for all $\mathrm{x}, \mathrm{y} \in \mathrm{M}$ and $\alpha \in \Gamma$.

Throughout the present paper we shall make extensive use of the following basic commutator identities: $[\mathrm{x} \beta \mathrm{y}, \mathrm{z}]_{\alpha}=\mathrm{x} \beta[\mathrm{y}, \mathrm{z}]_{\alpha}+[\mathrm{x}, \mathrm{z}]_{\alpha} \beta \mathrm{y}+\mathrm{x} \beta \mathrm{z} \alpha \mathrm{y}-\mathrm{x} \alpha \mathrm{z} \beta \mathrm{y}$

$[\mathrm{x}, \mathrm{y} \beta \mathrm{z}]_{\alpha}=\mathrm{y} \beta[\mathrm{x}, \mathrm{z}]_{\alpha}+[\mathrm{x}, \mathrm{y}]_{\alpha} \beta \mathrm{z}+\mathrm{y} \alpha \mathrm{x} \beta \mathrm{z}-\mathrm{y} \alpha \mathrm{x} \beta \mathrm{z}$

$(\mathrm{x} \beta \mathrm{y} \mathrm{oz})_{\alpha}=\mathrm{x} \beta(\mathrm{yoz})_{\alpha}-[\mathrm{x}, \mathrm{z}]_{\alpha} \beta \mathrm{y}+\mathrm{x} \beta \mathrm{z} \alpha \mathrm{y}-\mathrm{x} \alpha \mathrm{z} \beta \mathrm{y}=(\mathrm{xoz})_{\alpha} \beta \mathrm{y}+\mathrm{x} \beta[\mathrm{y}, \mathrm{z}]_{\alpha}+\mathrm{x} \alpha \mathrm{z} \beta \mathrm{y}-\mathrm{x} / / \mathrm{z} \alpha \mathrm{y}$, and

$(\mathrm{xo} y \beta z)_{\alpha}=(\text { xоy })_{\alpha} \beta \mathrm{z}-\mathrm{y} \beta[\mathrm{x}, \mathrm{z}]_{\alpha}+\mathrm{y} \alpha \mathrm{x} \beta \mathrm{z}-\mathrm{y} \beta \mathrm{x} \alpha \mathrm{z}=\mathrm{y} \beta(\mathrm{xoz})_{\alpha}+[\mathrm{x}, \mathrm{y}]_{\alpha} \beta \mathrm{z}+\mathrm{y} \beta \mathrm{x} \alpha \mathrm{z}-\mathrm{y} \alpha \mathrm{x} \beta \mathrm{z}$, for all $\mathrm{x}, \mathrm{y}, \mathrm{z} \in \mathrm{M}$ and $\alpha, \beta \in \Gamma$.

\section{Results}

In this paper we will take an assumption $(*) \ldots x \alpha y \beta z=x \beta y \alpha z$, for all $x, y \in M$ and $\alpha, \beta \in \Gamma$, and consider in all our results $\mathrm{M}$ satisfying this assumption. In order to prove our results, we need the following lemmas:

Lemma 3.1 [4]: For any $\Gamma$-derivation $D$ on a $\Gamma$-near-ring $M$. we have

(i) $(\mathrm{x} \gamma \mathrm{D}(\mathrm{y})+\mathrm{D}(\mathrm{x}) \gamma \mathrm{y}) \mu \mathrm{z}=\mathrm{x} \gamma \mathrm{D}(\mathrm{y}) \mu \mathrm{z}+\mathrm{D}(\mathrm{x}) \gamma \mathrm{y} \mu \mathrm{z}$, for all $\mathrm{x}, \mathrm{y}, \mathrm{z} \in \mathrm{M}$ and $\gamma, \mu \in \Gamma$.

(ii) $(\mathrm{D}(\mathrm{x}) \gamma \mathrm{y}+\mathrm{x} \gamma \mathrm{D}(\mathrm{y})) \mu \mathrm{z}=\mathrm{D}(\mathrm{x}) \gamma \mathrm{y} \mu \mathrm{z}+\mathrm{x} \gamma \mathrm{D}(\mathrm{y}) \mu \mathrm{z}$, for all $\mathrm{x}, \mathrm{y}, \mathrm{z} \in \mathrm{M}$ and $\gamma, \mu \in \Gamma$.

Lemma 3.2: Suppose that $D$ is a $\Gamma$-derivation on $\Gamma$-near-ring $M$. Then $M$ satisfies the 
following left distributive laws:

(i) $z \beta(x \alpha D(y)+D(x) \alpha y)=z \beta x \alpha D(y)+z \beta D(x) \alpha y$, for all $x, y, z \in M$ and $\alpha, \beta \in \Gamma$.

(ii) $\mathrm{z} \beta(\mathrm{D}(\mathrm{x}) \alpha \mathrm{y}+\mathrm{x} \alpha \mathrm{D}(\mathrm{y}))=\mathrm{z} \beta \mathrm{D}(\mathrm{x}) \alpha \mathrm{y}+\mathrm{z} \beta \mathrm{x} \alpha \mathrm{D}(\mathrm{y})$, for all $\mathrm{x}, \mathrm{y}, \mathrm{z} \in \mathrm{M}$ and $\alpha, \beta \in \Gamma$.

Proof: By definition of $\Gamma$-derivation D,and by lemma 3.1 .

Lemma 3.3 [4]: Let $M$ be a prime $\Gamma$-near-ring and let $U(\neq\{0\})$ be a right(resp. left) invariant subset of $M$. If $x$ is an element of M such that $U \Gamma x=\{0\}$ (resp. $x \Gamma U=\{0\}$ ), then $x=0$.

Lemma 3.4 [4]: Let $M$ be prime $\Gamma$-near-ring and let $U(\neq\{0\})$ be an invariant subset of $M$. If $D$ is a nonzero

$\Gamma$-derivation on $\mathrm{M}$, then for any $\mathrm{x}, \mathrm{y} \in \mathrm{M}$

(i) $\mathrm{x} \Gamma \mathrm{U} \Gamma \mathrm{y}=\{0\}$ implies $\mathrm{x}=0$ or $\mathrm{y}=0$,

(ii) $\mathrm{D}(\mathrm{U}) \Gamma \mathrm{y}=\{0\}$ implies $\mathrm{y}=\{0\}$,

Theorem 3.5: Let $M$ be prime $\Gamma$-near-ring, and let $U(\neq\{0\})$ be an invariant subset of $M$. If $M$ admits a generalized $\Gamma$-derivation $F$ associated with a non-zero $\Gamma$-derivation $D$ such that $F\left([u, v]_{\alpha}\right)= \pm[u, v]_{\alpha}$, for all $\mathrm{u}, \mathrm{v} \in \mathrm{U}$ and $\alpha \in \Gamma$, then $\mathrm{M}$ is commutative.

Proof: For all $\mathrm{u}, \mathrm{v} \in \mathrm{U}$ and $\alpha \in \Gamma$, let we have

$$
\mathrm{F}\left([\mathrm{u}, \mathrm{v}]_{\alpha}\right)=[\mathrm{u}, \mathrm{v}]_{\alpha}
$$

Replacing v with $\mathrm{u} \beta \mathrm{v}(1)$ and using it, we get

$$
\mathrm{D}(\mathrm{u}) \beta[\mathrm{u}, \mathrm{v}]_{\alpha}=0 \ldots \ldots \ldots \ldots \ldots \ldots \ldots(2) \text {, for all } \mathrm{u}, \mathrm{v} \in \mathrm{U} \text { and } \alpha, \beta \in \Gamma \text {. }
$$

Again for some $y \in M$, replacing $v$ with v $\delta y$ in (2) and using it, we obtain

that is,

$$
\mathrm{D}(\mathrm{u}) \beta \mathrm{v} \delta[\mathrm{u}, \mathrm{y}]_{\alpha}=0 \ldots \ldots \ldots \ldots \ldots \ldots . . . . . . . .(3) \text {, for all } \mathrm{u}, \mathrm{v} \in \mathrm{U} \text { and } \alpha, \beta, \delta \in \Gamma \text {. }
$$

$\mathrm{D}(\mathrm{u}) \Gamma \mathrm{U} \Gamma[\mathrm{u}, \mathrm{y}]_{\alpha}=0$.

It follows from Lemma 3.4 that either $\mathrm{D}(\mathrm{u})=0$ or $[\mathrm{u}, \mathrm{y}]_{\alpha}=0$, for all $\mathrm{u} \in \mathrm{U}, \mathrm{y} \in \mathrm{M}$ and $\alpha \in \Gamma$. Since $\mathrm{D} \neq 0$, therefore,

$$
[\mathrm{u}, \mathrm{y}]_{\alpha}=0 \ldots \ldots \ldots \ldots \ldots \ldots \ldots \ldots . .(4), \text { for all } \mathrm{u} \in \mathrm{U}, \mathrm{y} \in \mathrm{M} \text { and } \alpha \in \Gamma \text {. }
$$

For some $\mathrm{x} \in \mathrm{M}$, replacing u with $\mathrm{x} \gamma \mathrm{u}$ in (4) and using it, we obtain

that is,

$[\mathrm{x}, \mathrm{y}]_{\alpha} \gamma \mathrm{u}=0$, for all $\mathrm{x}, \mathrm{y} \in \mathrm{M}$, and $\alpha, \gamma \in \Gamma$.

$[\mathrm{x}, \mathrm{y}]_{\alpha} \Gamma \mathrm{U}=0$, for all $\mathrm{u} \in \underline{\mathrm{U}}$.

Then by lemma 3.3 for all $\mathrm{x}, \mathrm{y} \in \mathrm{M}$ and $\alpha \in \Gamma$, we have $[\mathrm{x}, \mathrm{y}]_{\alpha}=0$. Hence $\mathrm{M}$ is commutative .

Theorem 3.6: Let $M$ be prime $\Gamma$-near-ring, and let $U(\neq\{0\})$ be an invariant subset of $M$. If $M$ admits a generalized $\Gamma$-derivation $F$ associated with a non-zero $\Gamma$-derivation $D$ such that $F\left((\text { uov })_{\alpha}\right)= \pm$ (uov) ${ }_{\alpha}$, for all $\mathrm{u}, \mathrm{v} \in \mathrm{U}$ and $\alpha \in \Gamma$, then $\mathrm{M}$ is commutative.

Proof: For all $\mathrm{u}, \mathrm{v} \in \mathrm{U}$ and $\alpha \in \Gamma$, let we have

$$
\mathrm{F}\left((\text { uov })_{\alpha}\right)=(\text { uov })_{\alpha}
$$

Replacing $\mathrm{v}$ with $\mathrm{v} \beta \mathrm{u}(1)$ and using it, we get

$$
\text { (uov) } \alpha \beta \mathrm{D}(\mathrm{u})=0 \ldots \ldots \ldots \ldots \ldots \ldots .(2) \text {, for all } \mathrm{u}, \mathrm{v} \in \mathrm{U} \text { and } \alpha, \beta \in \Gamma \text {. }
$$

Again for some $\mathrm{y} \in \mathrm{M}$, replacing $\mathrm{v}$ with $\mathrm{y} \delta \mathrm{v}$ in (2) and using it, we obtain

$$
[\mathrm{u}, \mathrm{y}]_{\alpha} \delta \vee \beta \mathrm{D}(\mathrm{u})=0 \ldots \ldots \ldots \ldots \ldots . .(3) \text {, for all } \mathrm{u}, \mathrm{v} \in \mathrm{U} \text { and } \alpha, \beta, \delta \in \Gamma \text {. }
$$

Equation (3) is the same as (3) in the proof of Theorem 3.5.Thus, by using the same arguments as in the proof of Theorem 3.5. We can conclude the result here.

Theorem 3.7: Let $\mathrm{M}$ be a prime $\Gamma$-near-ring , and let $\mathrm{U}(\neq\{0\})$ be an invariant subset of $\mathrm{M}$. Suppose that $\mathrm{F}$ and $G$ be two generalized $\Gamma$-derivations of $M$ with associated $\Gamma$-derivations $D_{1}$ and $D_{2}$ respectively such that $(\mathrm{F}(\mathrm{u}) \alpha \mathrm{v}+\mathrm{F}(\mathrm{v}) \alpha \mathrm{u}) \pm(\mathrm{u} \alpha \mathrm{G}(\mathrm{v})+\mathrm{v} \alpha \mathrm{G}(\mathrm{u}))=0$, for all $\mathrm{u}, \mathrm{v} \in \mathrm{U}$ and $\alpha \in \Gamma$, or $[\mathrm{F}(\mathrm{u}), \mathrm{v}]_{\alpha} \pm[\mathrm{u}, \mathrm{G}(\mathrm{v})]_{\alpha}=0$, for all $\mathrm{u}, \mathrm{v} \in \mathrm{U}$ and $\alpha \in \Gamma$, then $\mathrm{M}$ is commutative. 
Proof: For all $\mathrm{u}, \mathrm{v} \in \mathrm{U}$ and $\alpha \in \Gamma$,let we have

$(\mathrm{F}(\mathrm{u}) \alpha \mathrm{v}+\mathrm{F}(\mathrm{v}) \alpha \mathrm{u}) \pm(\mathrm{u} \alpha \mathrm{G}(\mathrm{v})+\mathrm{v} \alpha \mathrm{G}(\mathrm{u}))=0$

Combining the expressions obtained after replacing $\mathrm{u}$ by $\mathrm{u} \beta \mathrm{v}$ in $(1)$ and multiplying(1) with $\mathrm{v}$ from the right, we get

$$
\mathrm{u} \beta \mathrm{D}_{1}(\mathrm{v}) \alpha \mathrm{v}+\mathrm{v} \alpha \mathrm{u} \beta \mathrm{D}_{2}(\mathrm{v})+\mathrm{u} \beta[\mathrm{v}, \mathrm{G}(\mathrm{v})]_{\alpha}=0 \ldots \ldots \ldots .(2), \text { for all } \mathrm{u}, \mathrm{v} \in \mathrm{U} \text { and } \alpha, \beta \in \Gamma
$$

For any $y \in M$, replacing $\mathrm{u}$ by $y \delta u$ in (2) and combining with the expression obtained by multiplying (2) with $y$ from the left, we get

$$
[\mathrm{v}, \mathrm{y}]_{\alpha} \delta \mathrm{u} \beta \mathrm{D}_{2}(\mathrm{v})=0
$$$$
\text { (3), for all } \mathrm{u}, \mathrm{v} \in \mathrm{U} \text { and } \alpha, \beta, \delta \in \Gamma \text {. }
$$

For any $z \in M$, replacing $u \beta \mathrm{D}_{2}(\mathrm{v})$ by $z \delta \mathrm{u} \beta \mathrm{D}_{2}(\mathrm{v})$ in (3), we obtain

$$
[\mathrm{v}, \mathrm{y}]_{\alpha} \Gamma \mathrm{M} \Gamma \mathrm{U}=0
$$

Since $U \neq\{0\}$ and $M$ is a prime, we obtain $[v, y]_{\alpha}=0$

Replacing $\mathrm{v}$ by $\mathrm{x} \gamma \mathrm{v}$ in (4) and using it,we obtain

$$
[\mathrm{x}, \mathrm{y}]_{\alpha} \gamma \mathrm{v}=0 \text {, for all } \mathrm{x}, \mathrm{y} \in \mathrm{M} \text {, and } \alpha, \beta \in \Gamma \text {. }
$$

Then by lemma 3.3 for all $v \in U$, we have $[x, y]_{\alpha}=0$, for all $\mathrm{x}, \mathrm{y} \in \mathrm{M}$, and $\alpha \in \Gamma$.

Hence $\mathrm{M}$ is commutative.

(ii) Similarly we can prove that $\mathrm{M}$ is commutative, if $[\mathrm{F}(\mathrm{u}), \mathrm{v}]_{\alpha} \pm[\mathrm{u}, \mathrm{G}(\mathrm{v})]_{\alpha}=0$ satisfied, for all $\mathrm{u}, \mathrm{v} \in \mathrm{U}$ and $\alpha \in \Gamma$.

Remark 3.8: Taking $\mathrm{G}=\mathrm{F}$ or $\mathrm{G}=-\mathrm{F}$ in the hypothesis of Theorem 3.7, we get the following corollary.

Corollary 3.9: Let $M$ be a prime $\Gamma$-near-ring , and let $U(\neq\{0\})$ be an invariant subset of $M$. Suppose that $F$ and $\mathrm{G}$ be two generalized $\Gamma$-derivations of $\mathrm{M}$ with associated $\Gamma$-derivations $\mathrm{D}_{1}$ and $\mathrm{D}_{2}$ respectively such that $[\mathrm{F}(\mathrm{u}), \mathrm{v}]_{\alpha}+[\mathrm{F}(\mathrm{v}), \mathrm{u}]_{\alpha}=0$, for all $\mathrm{u}, \mathrm{v} \in \mathrm{U}$ and $\alpha \in \Gamma$, or $(\mathrm{F}(\mathrm{u}) \circ \mathrm{v})_{\alpha}+\left(\mathrm{F}(\mathrm{v})^{\circ} \mathrm{u}\right)_{\alpha}=0$, for all $\mathrm{u}, \mathrm{v} \in \mathrm{U}$ and $\alpha \in \Gamma$, then $\mathrm{M}$ is commutative.

Theorem 3.10: Let $M$ be a 2-torsion free prime $\Gamma$-near-ring , and $U(\neq\{0\})$ be an invariant subset of $M$. Suppose that $F$ and $G$ be two generalized $\Gamma$-derivations of $M$ with associated $\Gamma$-derivations $D_{1}$ and $D_{2}$ respectively such that $\mathrm{F}\left([\mathrm{u}, \mathrm{v}]_{\alpha}\right)=[\mathrm{F}(\mathrm{u}), \mathrm{v}]_{\alpha}+[\mathrm{D}(\mathrm{v}), \mathrm{u}]_{\alpha}$, for all $\mathrm{u}, \mathrm{v} \in \mathrm{U}$ and $\alpha \in \Gamma$, or $(\mathrm{F}(\mathrm{u}) \circ \mathrm{v})_{\alpha}-\left(\mathrm{D}(\mathrm{v})^{\circ} \mathrm{u}\right)_{\alpha}=0$, for all $\mathrm{u}, \mathrm{v} \in \mathrm{U}$ and $\alpha \in \Gamma$, then $\mathrm{M}$ is commutative.

Proof: By our hypothesis, we have

$\mathrm{F}\left([\mathrm{u}, \mathrm{v}]_{\alpha}\right)=[\mathrm{F}(\mathrm{u}), \mathrm{v}]_{\alpha}+[\mathrm{D}(\mathrm{v}), \mathrm{u}]_{\alpha} \ldots \ldots \ldots . .(1)$, for all $\mathrm{u}, \mathrm{v} \in \mathrm{U}$ and $\alpha \in \Gamma$

Replacing $\mathrm{v}$ by $\mathrm{v} \beta \mathrm{u}$ in (1) and employing (1),we find that

$$
2[\mathrm{u}, \mathrm{v}]_{\alpha} \beta \mathrm{D}(\mathrm{u})=\mathrm{v} \beta[\mathrm{F}(\mathrm{u}), \mathrm{u}]_{\alpha}+\mathrm{v} \beta[\mathrm{D}(\mathrm{u}), \mathrm{u}]_{\alpha} \ldots \ldots(2) \text {, for all } \mathrm{u}, \mathrm{v} \in \mathrm{U} \text { and } \alpha, \beta \in \Gamma
$$

For any $r \in M$, putting $\mathrm{v}$ by $\mathrm{r} \delta \mathrm{v}$ in (2) and applying (2), we get

$$
2[\mathrm{u}, \mathrm{r}]_{\alpha} \delta \mathrm{v} \beta \mathrm{D}(\mathrm{u})=0 \text {. }
$$

Since $\mathrm{M}$ is 2-torsion free, we get $[\mathrm{u}, \mathrm{r}]_{\alpha} \delta \mathrm{v} \beta \mathrm{D}(\mathrm{u})=0, \ldots \ldots \ldots .(3)$, for all $\mathrm{u}, \mathrm{v} \in \mathrm{U}, \mathrm{r} \in \mathrm{M}$ and $\alpha, \beta, \delta \in \Gamma$.

Equation (3) is the same as (3) in the proof of Theorem 3.5, Thus, by using the same arguments as in the proof of Theorem 3.5, we can conclude the result here.

Similar arguments can be adapted in the case $(\mathrm{F}(\mathrm{u}) \circ \mathrm{v})_{\alpha}-\left(\mathrm{D}(\mathrm{v})^{\circ} \mathrm{u}\right)_{\alpha}=0$, for all $\mathrm{u}, \mathrm{v} \in \mathrm{U}$ and $\alpha \in \Gamma$, and we can omit the same proof.

Theorem 3.11: Let $M$ be a prime $\Gamma$-near-ring , and let $U(\neq\{0\})$ be an invariant subset of $M$. Suppose that $F$ and $G$ be two generalized $\Gamma$-derivations of $M$ with associated $\Gamma$-derivations $D_{1}$ and $D_{2}$ respectively such that $F\left(u^{2}\right) \pm$ $\mathrm{u}^{2}=0$, for all $\mathrm{u} \in \mathrm{U}$, or $\mathrm{D}(\mathrm{u}) \alpha \mathrm{F}(\mathrm{v}) \pm \mathrm{u} \alpha \mathrm{v}=0$, for all

$\mathrm{u}, \mathrm{v} \in \mathrm{U}$ and $\alpha \in \Gamma$, then $\mathrm{M}$ is commutative.

Proof: From the hypothesis, let

(i) $F\left(u^{2}\right)=u^{2}$, for all $u \in U$.

Replacing $u$ by $u+v$ in (1), and using it, we obtain

$\mathrm{F}\left((\mathrm{uov})_{\alpha}\right)=(\mathrm{uov})_{\alpha}$, for all $\mathrm{u}, \mathrm{v} \in \mathrm{U}$ and $\alpha \in \Gamma$.

Using Theorem 2.6, we get the required result.

(ii) $\mathrm{F}\left(\mathrm{u}^{2}\right)+\mathrm{u}^{2}=0$, for all $\mathrm{u} \in \mathrm{U}$, then as (i) we get 
$\mathrm{F}\left((\text { uov })_{\alpha}\right)+(\text { uov) })_{\alpha}=0$, for all $\mathrm{u}, \mathrm{v} \in \mathrm{U}$ and $\alpha \in \Gamma$.

Following the same technique as used in the proof of Theorem 3.6, we get the required result.

Corollary 3.12: Let $M$ be a prime $\Gamma$-near-ring , and let $U(\neq\{0\})$ be an invariant subset of $M$. Suppose that $F$ and $\mathrm{G}$ be two generalized $\Gamma$-derivations of $\mathrm{M}$ with associated $\Gamma$-derivations $\mathrm{D}_{1}$ and $\mathrm{D}_{2}$ respectively such that $[\mathrm{F}(\mathrm{u}), \mathrm{v}]_{\alpha}=[\mathrm{u}, \mathrm{F}(\mathrm{v})]_{\alpha}$, for all $\mathrm{u}, \mathrm{v} \in \mathrm{U}$ and $\alpha \in \Gamma$, or $[\mathrm{F}(\mathrm{u}), \mathrm{v}]_{\alpha}+[\mathrm{u}, \mathrm{F}(\mathrm{v})]_{\alpha}=0$, for all $\mathrm{u}, \mathrm{v} \in \mathrm{U}$ and $\alpha \in \Gamma$, then $\mathrm{M}$ is commutative.

\section{References}

[1]. M. Asci, $\Gamma-(\sigma, \tau)$-derivation on gamma near ring, International Math.Forum 2, No.3(2007), 97-102.

[2]. Y. U. Cho and Y. B. Jun. Gamma-derivations in prime and semiprime gamma-near rings.

[3]. Indian J. Pure Appl. Math., 33(10),(2002),1489-1494.

[4]. Y.U. Cho, Some conditions on derivations in prime near rings, J. Korea Soc. Math. Educ., Ser. B, Pure Appl. Math., 8, No. 2 (2001), 145-152.

[5]. Y. B. Jun, K. H. Kim, and Y. U. Cho, On gamma-derivations in gamma near-rings, Soochow J. Math. 29 (2003), no. 3, $275-282$.

[6]. M. Kazaz, A. Alkan, Two sided $\Gamma$ - $\alpha$-derivations in prime and semiprime $\Gamma$-near-rings, Commun. Korean Math. Soc., 23, No. 4 (2008), 469-477.

[7]. B. Satyanarayana, A Note on $\Gamma$ - Near Rings, Indian J. of Math. 41(3), (1999), 427-433.

[8]. M. Uckun, M.A. Ozturk, Y.B. Jun, On prime gamma-near-rings with derivations, Commun. Korean Math.

[9]. Soc., 19, No. 3 (2004), 427-433. 